\title{
Chapter 1 \\ Wearable Sensors for Gesture Analysis in Smart Healthcare Applications
}

\author{
Abdul Haleem Butt*, Alessandra Moschetti*, Laura \\ Fiorini, Paolo Dario, Filippo Cavallo
}

*equal contribution

\subsection{Introduction: healthcare and technology}

The population of the EU is expected to reach 526 million by 2050 , and the demographic old-age dependency ratio (number of people age 65 or above compared to those age 15-64) projected to rise from the current $28 \%$ to $50 \%$ by 2060 [4]. This evident demographic shift foretells that understanding healthy aging and age-related diseases will be a future challenge. Additionally, the decrease of working-age population will lead to an increase in the demand for nurse practitioners (+94\% in 2025) [16] and physicians assistants (+72\% in 2025) [37], as well as an increased need for a higher level of care and for future assistance. Today, having a good quality of care and highly sustainable health-care services are increasingly imperative in EU countries citerobotfuture 2016.

Several research efforts conducted in recent years highlight the primary needs of elderly people and stakeholders [1][2]. Elderly citizens suffer from cognitive and physical disorders due to natural decline, and mental and physical impairments [2]. People with cognitive disorders could have problems in keeping control of their lives, and in effect, recent studies correlate changes in the daily behavior of older persons with cognitive problems [25]. Additionally, older individuals need to reduce the risk of accidents at home and may require assistance in managing chronic diseases [1]. Due to all these reasons, over the past few years, requests for home-care services have increased [72].

The main objective of Ambient Assisted Living (AAL) is to provide adequate technological solutions which could increase the level of quality of life [56]. AAL applications span both helping the different stakeholders in preventing accidents and monitoring elderly people at home [75]. Recent advances in mobile internet, the Internet of Things (IoT), and telehealth services increase the perception abilities of the devices, thus providing an efficient "Continuum of Care" and assistance everywhere. The users will be monitored constantly, without the necessity for a person to 
live with them [79]. In effect, a recent study [34] underlines that the improvement in the management of health conditions, remote feedback from nurses, and feeling of safety and self-confidence about their status are all improving the use and acceptance of telehealth technologies.

The understanding of human movements, behavior, and body language is of obvious importance in AAL applications. It can assist in using technological solutions for prevention or monitoring and can also help the communication between the users and the technological devices.

This chapter presents a general overview of the importance of the recognition of human gestures to deliver personalized and efficient services to promote the independent living of elderly individuals. After a brief introduction about smart sensors (Section 1.2) and gesture recognition applications in AAL (Section 1.3), the authors describe the primary technologies used to capture the hand motion (Section 1.4), data processing, and classification algorithms used in gesture recognition (Section 1.5). Additionally, the authors present two concrete applications of gesture recognition (Section 1.6).

\subsection{Growth of smart sensors, wearables, and IoT}

The rising demand for sustainable healthcare systems has increased the importance of AAL developments, services, and products. The market for medical electronics is expected to reach USD 4.41 billion by 2022 [6], whereas the smart home market, which has been experiencing steady growth, is expected to reach USD 121.73 billion by 2022 [7].

Similarly, the telehealth market was valued at $\$ 2.2$ billion in 2015 and is predicted to reach $\$ 6.5$ billion by 2020 , with an annual growth rate of $24.2 \%$ [8]. The home healthcare industry is also testing tele-homecare and tele-monitoring services that represent a valuable opportunity to balance quality of care with cost control. In effect, according to [34], the possibility to be monitored continuously and the evidence of reduced costs are considered some of the facilitators of telehealth. In this manner, caregivers and family could be connected constantly by smartphone, tablet, or other connected device.

According to [9], more than 10,000 healthcare apps are available in the Apple/Google store. These statistics also reveals that approximately $85 \%$ of doctors use smartphones and medical apps, and $80 \%$ of them would like their patients to monitor their health status at home. Moreover, the digital impact on userexperience is clear, as more than $78 \%$ of users are interested in mobile-health solutions.

The number of worldwide mobile subscriptions was equal to 7.3 billion in 2015 and is predicted to reach 9 billion in 2021 with an annual growth rate of $5 \%$. We are now living in the IoT era, where not only are people connected to internet, but also the number of connected devices was equal to 15 billion in 2015 and is expected to reach 28 billion in 2021 with an annual growth rate of $27 \%$ [5].

Wearable devices are now coming to market with form factors that increase comfort during various daily activities. CCS Insight has updated its outlook on the future of wearable tech, indicating that it expects 411 million smart wearable devices to be 
sold in 2019 and that fitness and activity trackers will account for more than $50 \%$ of the unit sales. Particularly, smartwatches will account for almost half of wearables revenue in 2019 [3].

All these smart devices can provide a significant amount of information that could be used in the AAL context to support the understanding of human movements, behavior, and body language, without being invasive, but simply as a part of daily living.

\subsection{Application scenarios}

Recognition of human movements and gestures plays an important role in AAL solutions. Using them, caregivers and family could monitor and assist elderly persons. In particular, different scenarios can be identified that span from human-robot interaction (HRI) to monitoring applications, as shown in Fig. 1.1

\section{Gesture recognition in human-robot interaction}

One of the challenges in HRI is make the robot understand human behavior as it occurs in human-human interaction. Thus, the robot could be able to perceive the action or intention of the user without the person communicating directly to the robot. Inter-personal communication includes non-verbal cues, such as facial expressions, movements, and hand gestures that are used to express feeling and give feedback. Having the abilities to sense and respond appropriately to the users is important to tailor the content of the interaction, increasing the robots social abilities [79]. The robot could be able to perceive what the user is doing, whether he is eating or drinking or talking to somebody, or simply alone on the sofa becoming bored; in this way, it could approach the user in a tailored manner and attempt to interact properly.

\section{Prevention of physical and cognitive degeneration}

Typical AAL scenarios that involve the use of gesture recognition systems are the ones that aim at preventing early degeneration or dangerous situations by stimulating cognitive and physical abilities [56]. AAL solutions can help elderly people by providing personalised games, made also of different sensors that can detect human movements. In this way, elderly individuals, and others as well, can perform special games that are enjoyable and also provide assistance with physical and cognitive exercises. Through recognition of human movements, caregivers will be able to determine if the person is doing the movement correctly. In this way, people will be able to perform exercises and activities while being monitored by experts. Thanks to these applications, elderly people will be able to train themselves and keep themselves fit, decreasing the risk of degeneration of physical and cognitive abilities [1]. 


\section{Gesture recognition in monitoring applications}

Recognition of movements and gestures plays an important role in monitoring applications, as well. This recognition can assist both in monitoring daily activities and in rehabilitation purposes. Elderly people living alone can change their habits, and this can be seen as a first symptom of a degeneration in cognitive abilities. Often, people with dementia forget to eat and drink or to carry out simple activities of daily living such as personal hygiene behaviors [25]. Having the ability to recognize daily gestures would allow remote monitoring of elderly people to determine whether people can still maintain their daily routines [70]. Alternatively, many persons, and in particular, the elderly, could have to perform exercises at home as assessment tools or for rehabilitation purposes. In this case, the recognition of movements and gestures could help in enabling a continuum of care. After the first training sessions accomplished with the therapist, the patient could perform the activities at home while being monitored. In this way, the user could increase the amount of sessions spent doing the exercises, without the need for a session with the physician [2].

\section{Gesture recognition to control robots and smart appliances}

Gesture recognition could also be used to control smart appliances, thanks to the recognition of different movements [2]. These recognition abilities could be used to interact with robots or other devices to enable the people to work longer or continue engaging in personal hobbies and activities that can become difficult to perform after a certain age. Concerning the work, AAL solutions can be used to compensate for sensory and motor deficits, preventing work-related injuries as well. To maneuver heavy or large objects, a remotely controlled robotic arm could be used, instead of a joystick or mouse, by recognizing the gesture the worker is making [2].

\subsection{Gesture recognition technology}

The motion capture of human gestures is quite complex due to independent movements of the five fingers [60]. Thus, the technologies related to the capture of gestures should be developed to be fully reliable and highly sensitive, with a low level of invasiveness to minimize the discomfort of the monitoring task. In recent years, many devices based on cameras and inertial sensors have been created to recognize nonverbal behaviors that are important in human communication [35]. Some of them are research products, and others are available on the market. However, most of the developed systems present strong limitations. For instance, regarding cameras, the system based on the three-dimensional (3-D) infrared camera for finger recognition, or other devices based on camera recognition, suffer from line-of-sight obstructions and have heavy computation requirements [31]. Recent research based on 3-D depth 

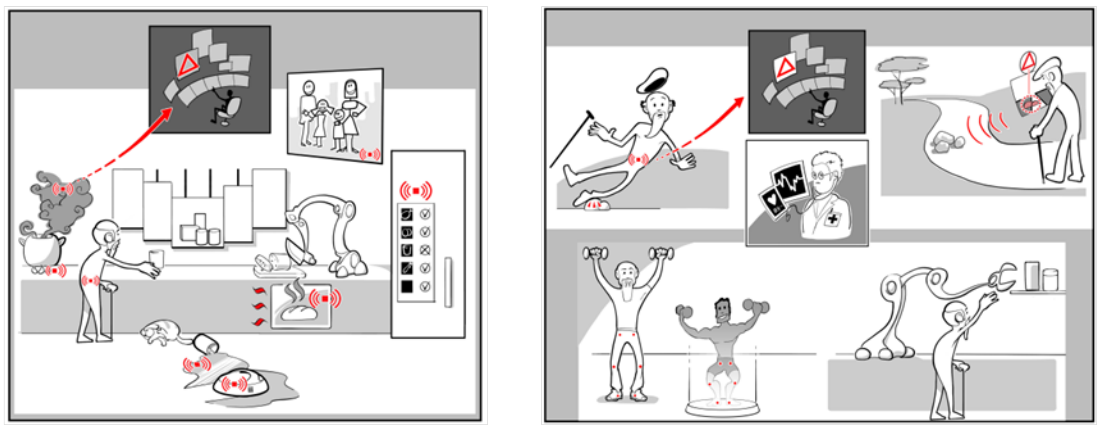

Figure 1.1: Example of AAL scenarios

sensors, such as the Leap Motion controller (LMC) and Microsoft Kinetic sensor demonstrate a high degree of segmentation and 3-D hand gesture recognition [49].

In contrast, wearable sensors overcome the limitation of obstruction and light. Being worn by the user, they receive information directly from the movement of the user. However, to use this type of sensor, the user has to wear something on the hand, which can be felt as cumbersome and can limit the ability to perform gestures in a natural way [52]. However, thanks to the miniaturization and affordability of these sensors, particularly of Inertial Measurement Units (IMUs), wearable sensors have demonstrated good potential in recognizing activities [45]. The combination of different types of sensors can improve the accuracy of recognition tasks, but in the case of wearable sensors, it is important to always pay attention to obtrusiveness, maintaining a good trade-off between recognition performance and invasiveness [54].

In this section, some technological solutions, also commercially available, are introduced and described to provide a general overview of the current solutions used for hand gesture recognition.

\subsubsection{SensHand}

The SensHand is a device developed by Cavallo et al. [22] [29] in 2013. This device is composed of four, nine-axis inertial sensor units to be worn on the hand as rings and bracelet. In particular, three modules are designed to be worn on three fingers (typically thumb, index, and middle finger), and one module is designed to be worn on the wrist.

The SensHand has gone through different improvements over the years (see Fig 1.2. The last version is made of four inertial sensors integrated into four INEMOM1 boards with dedicated STM32F103xE family microcontrollers (ARM 32-bit Cortex-M3 CPU, from STMicroelectronics, Milan, Italy). Each module includes a LSM303DLHC (six-axis geomagnetic module, dynamically user-selectable full scale acceleration, and a magnetic field, from STMicroelectronics, Milan, Italy), a 
L3G4200D (three-axis digital gyroscope, user-selectable angular rate, from STMicroelectronics, Milan, Italy), and I2C digital output. The integration of these sensors enables three-dimensional mapping of the motion.

A Controller Area Network standard is used to implement module coordination and data synchronization. The module placed on the wrist is the coordinator of the system: it collects data and transmits them through the Bluetooth V3.0 communication protocol via the SPBT2632C1A (STMicroelectronics, Milan, Italy) Class 1 module toward a generic control station. A small, rechargeable, and light Li-Ion battery supplies power to the device. This battery is integrated in the coordinator module. A fourth-order low-pass digital filter with a cutoff frequency of $5 \mathrm{~Hz}$ has been implemented and can be used to remove high-frequency noise and tremor frequency bands in real time [66]. Data are collected on a PC by means of a custom interface developed in C\# language. This interface, beyond collecting the data, allows selection of a low-pass filter if desired and the acquisition frequency. Data can be sent at $100 \mathrm{~Hz}$ or $50 \mathrm{~Hz}$, depending on the application.

The interface, together with embedded firmware, allows calibration of the three sensors by following some simple rules indicated by the interface (that follows the indication given by the manufacturer of the sensors). In this way, it is possible to calibrate the sensors easily, without reprogramming the device, and removing offsets and sensitivity that can affect the measurements.

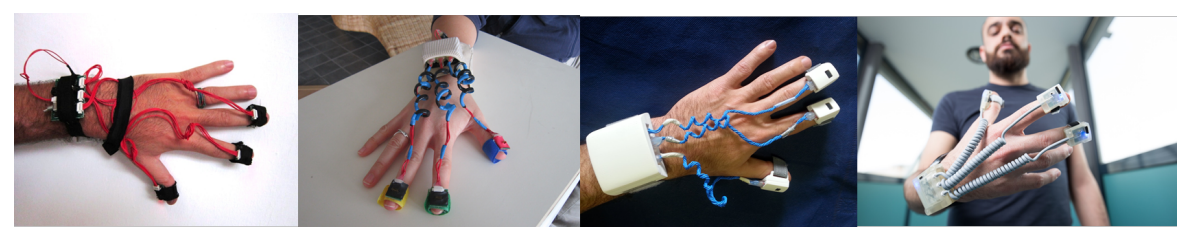

Figure 1.2: Different version of the SensHand

\subsubsection{Other Gloves}

Over the past 30 years or more, researchers have begun developing wearable devices, particularly glove-based systems, to recognize hand gestures. Different working principles have been studied during these years to find a good trade-off between precision, accuracy, and obtrusiveness [27].

\section{Glove-based system}

Much attention has been given to glove-based systems because of the natural fit of developing something to be worn on the hand to measure hand movement and finger bending. A glove-based system can be defined as: a system composed of an array of sensors, electronics for data acquisition/processing and power supply, and a support for the sensors that can be worn on the users hand [27]. The various devices can differ based on sensor technologies (i.e. piezoresistive, fiber optic, Hall effect, etc.), number of sensors per finger, sensor support (i.e. cloth or mechanical 
support), sensor location (i.e. hand joints, fingertip positions, etc.), and others [27]. A typical example of a glove-based system is the CyberGlobe, a cloth device with 18 or 22 piezo-resistive sensors that measures the flexion and the abduction/adduction of the hand joints (depending on the number of sensors, the measurable movements increase). It is considered one of the most accurate commercial systems [27] and has provided good results in recognizing sign language based on the biomechanical characteristics of the movement of the hand [61] [53]. CyberGlove showed good results also in applications of robot control and 3-D modelling [47].

Another example of a glove-based system is the 5DT Glove, which is based on optical fiber flexor sensors. The bending of the fingers is measured by measuring the intensity of the returned light indirectly [27]. Each finger has a sensor that measures the overall flexion of the finger. This device is famous for application in virtual reality [41], [47].

Other examples of glove-based systems used for sign language include clothsupported bend sensors based on the Hall Effect mounted together with an accelerometer [24] as well as flex and contact sensors [64]. Carbonaro et al. [21] added textile electrodes and an inertial motion unit to the deformation sensors of the glove-based device to add emotion recognition made through electrodermal activity.

\section{IMU-based system}

Even though glove-based systems can be very accurate in measuring hand degrees of freedom, they also can be perceived as cumbersome by users because they reduce the dexterity and natural movements. To reduce the invasiveness of the wearable sensors, different technologies have been introduced to recognize hand and finger gestures. One of the proposed solutions introduced the use of IMUs to recognize gestures. In particular, these sensors are worn on the hand to perceive the movements made by the fingers and the hand. The SensHand technology previously described belongs to this category of sensor. Moreover, Bui et al. [20] used the AcceleGlove with an added sensor on the back of the hand to recognize postures in Vietnamese Sign Language. In this way, they were able to evaluate the angle between the fingers and the palm and use it for identifying the gesture made.

Kim et al. [44] developed a device composed of three 3-axis accelerometers, worn on two fingers and on the back of the hand to recognize gestures. With the help of these sensors, they reconstructed the kinematic chain of the hand, allowing the device to recognize simple gestures. In addition, an accelerometer and a gyroscope were used on the back of the hand by Amma et al. [14] to recognize 3-D-space handwriting gestures.

Lei et al. [46] implemented a wearable ring with an accelerometer to recognize 12 one-stroke finger gestures, extracting temporal and frequency features. The chosen gestures were used to control different appliances in the house [42].

Wearing two 3-axis accelerometers on the thumb and on the index finger, Hsieh et al. [39] were able to recognize some simple gestures made using these fingers. Using a nine-axis inertial measurement unit, Roshandel et al. [65] were able to recognize nine different gestures with four different classifiers.

\section{EMG-based and hybrid systems}


New sensors have been developed to read the surface electromyography (sEMG), which is the recording of the muscle activity from the surface, detectable by surface electrodes. In recent years, attention has been paid to this kind of signal to recognize hand and finger gestures. Various technical aspects have been investigated to determine the optimal placement of the electrodes, and select the optimal features for the more appropriate classifier [59]. Naik et al. [59] used sEMG to recognize the flection of the fingers, both alone and combined, obtaining good results (accuracy $<$ 0.84). Using the same signal, Jung et al. [43] developed a new device that uses air pressure sensors and air bladders to measure the EMG signal.

To increase the number and the kind of gestures to be recognized, often different sensors are combined together. Frequently, EMG is combined with inertial sensors worn on the forearm to increase the amount of information obtained by the device. Wolf et al. [78] presented the BioSleeve, a device made of sEMG sensors and an IMU to be worn on the forearm. By coupling these two types of sensor, the authors were able to recognize 16 hand gestures, corresponding to various finger and wrist positions that were then used to command robotic platforms. Lu et al. [?] uses a similar combination of sensors to recognize 19 predefined gestures to control a mobile phone, achieving average accuracy of 0.95 in user-dependent testing. Georgie et al. [33] fused the signals of an IMU worn on the wrist with EMG at the forearm to recognize a total of 12 hand and finger gestures with a recognition rate of 0.743 .

\subsubsection{Leap Motion}

The Leap Motion Controller (LMC), is a commercially available optical device that can detect the hand motion and position in the 3-D space. Its weigh is $45 \mathrm{~g}$ and it is composed of three light emitting diodes (LEDs) and two infrared (IR) cameras with depth sensing abilities (resolution of 640x240 each). Both IR cameras are a distance of $20 \mathrm{~mm}$ from the center of the LMC, as depicted in Fig 1.3 a. The field of view in the hemispherical area is approximately 150.

The information regarding the user's hand, fingers, and gestures is captured as long as the hand is between $25 \mathrm{~mm}$ and $600 \mathrm{~mm}$ above the center of the sensor. The hand position captured is relative to the center of LMC [69].

The Leap Motion software development kit (SDK) allows access of the data for hand direction, speed, and rotation, in time-dependent frames with the average frequency of $100 \mathrm{~Hz} / \mathrm{s}$, which is considered adequate to capture the movement of the human hand with a high degree of accuracy. For hand movement detection, LMC software uses an internal model of the human hand to estimate the position of the hand, fingers, and gestures, even if the hand is not visible. This model uses dedicated algorithms to collect the information from the visible part of the hand and past observations to calculate the most likely position [12]. LMC also is able to detect the motion of each finger through dedicated algorithms. This aspect represents one of the most important advantages of LMC, which make it more convenient for the different applications.

Leap motion provides two types of APIs to acquire the data from LMC: native interface and WebSocket interface $(1.3 \mathrm{~b}$ ). The native interface provides dy- 
namic loaded libraries (DLL). These libraries contain sets of classes and datastructure which allows collection and analysis of data from the device. These libraries can be connected directly with $\mathrm{C}++$ and Objective-C applications, or through one of the language bindings provided for Java, C\#, and Python [12].

The WebSocket interface allows the interaction with LMC through the web server and web browser. The communication protocol is hypertext transfer protocol (HTTP), and the messages are codified in JavaScript object notation messages (JSON) to be easy for humans to read and write [12].

\subsubsection{Smartwatch}

Smartwatches are commercial, wearable devices capable of measuring physiological parameters like heart rate variability and temperature and capable of estimating the movement of the wrist by means of three-axial accelerometers. These devices allow a continual monitoring of the users daily activities. The acquired information could be analyzed and used for different purposes such as wellness, safety, gesture recognition, and the detection of an activity/inactivity period [19]. Today, many different smartwatches are available on the market. Apple watch, Samsung Gear S3, Huawei Watch, and Polar M600 are several examples.

Because of the smartwatchs sensor functionality, the device enables new and innovative applications that expand the original application field. Since 2001 [62], researchers have been paying attention to the movement of the wrist to estimate hand gestures. Now, smartwatches are offering a concrete possibility to exploit the recognition of gestures in real applications. For instance, Wile et al. [76] use smartwatches to identify and monitor the Parkinson Diseases postural tremor in 41 subjects. The results suggest that the smartwatch could measure the tremor with good correlation with respect to other devices. In other research, Liu et al. [48] fuse the information of an inertial sensor placed on the wrist with a Kinect camera to identify different gestures (agree, question mark, shake hand) to improve the interaction with a companion robot. Ahanathapillai et al. [10] use an Android smartwatch to recognize common daily activities such as walking and ascending/descending stairs. The parameters that are extracted in this work include activity level and step count. The achievements confirm good recognition of all the selected features.

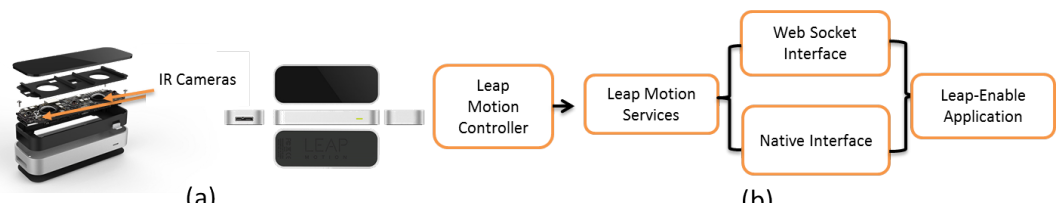

(a)

(b)

Figure 1.3: (a) Internal structure of Leap Motion Controller; (b) Leap Motion Controller system architecture 


\subsection{Description of the main approaches for gesture classification}

The data collected from the devices should be properly processed to extract significant features and then used to built a recognition model via machine learning techniques. This section describes the main features which could be extracted from lip motion devices and IMU glove (SensHand), and discusses the main techniques to select and classify the features adopted in AAL applications.

\subsubsection{Features used in gesture recognition for $A A L$}

To use the signals coming from the different devices, it is necessary to extract significant features, which means: " filtering relevant information and obtaining quantitative measures that allow signals to be compared" [45].

Several features could be extracted from data, depending on the technology used. In general, a statistical and a structural approach have been proposed to extract features from the time series. The former approach uses quantitative characteristics of the data (e.g. Fourier Transform) while the latter uses interrelationships among the data. Whether to use one or the other depends on the signal to be analyzed [45]. However, the selection of features depends primarily on the application of the gesture recognition system. In this context, the authors will present concrete applications of various sets of features in different AAL solutions.

As described in the previous section, LMC devices used depth imaging to detect the hand motion by using a hand skeletal model. Furthermore, appropriate depth segmentation techniques, based on a computer vision algorithms, are able to reconstruct hand motion, even if the full hand is not completely visible [58]. By default, LMC provides the time-domain features from captured hand movements and can recognize four different gestures.

The time-domain features include the physical properties of the hand, arm, and fingers; motions like velocity, speed, distance, angle (pitch, roll, yaw), position (x,y,z), length, and width; grab strength (hand opening and closing strength); and pinch strength (colliding strength between fingers).

The recognized gestures are screen tap, key tap, swipe, and circle; they are used to access specific movement patterns. The movement parameters provided by LMC for circle gesture are minimum radius $(\mathrm{mm})$ and minimum arc length (rad). For swipe gesture, the parameters are minimum velocity and minimum length. Finally, for screen tap and key tap, the extracted features are minimum velocity and finger movements.

However, some problems were experienced with the LMC. The device cannot detect the fingertip parameters when two (or more) fingers are very close to each other or the hand is in neutral position. The LMC will stop detecting the hands and fingers if the hands are tilted and the fingers are no longer in the field of view of the device. Additionally, LMC often loses the focus during the hand motion, which makes the features extraction procedures unreliable.

As confirmed from the state of the art, LMC gesture recognition is used in the different rehabilitation games. Exploiting the gamification paradigm [26], it has been 
introduced in the rehabilitation therapy of stroke patients to motivate them to pull, push, and grasp.

LMC is also often used to estimate the essential tremor in aging people and in patients with Parkinsons disease (PwPD). The traditional clinical assessment is still lacking in automated procedures able to quantify the severity of the hand tremor. In this context, LMC could represent a valid tool which could be used by clinicians. It is able to detect high-frequency movements such as tremor of the fingers and hand. Chen et al. [23] showed that the amplitude measured by LMC presents a strong linear correlation with the amplitude revealed by an accelerometer. In [38], LMC was used to overcome the limitations of the Kinetic sensor to assess the small movements of the PwPD hand such as rest tremor and postural tremor.

Lu et al. [50] used hand palm direction, fingertip positions, palm center position, and other relevant points to track the dynamic movements of daily activities such as opening and closing the hand, movement of fingers, hand movement in circle, and finger movements in upward or downward direction without requiring extra computation. Moreover, in the same study, other features were extracted from the hand gesture: Poke, Pinch, Pull, Scrape, Slap, Press, Cut, Circle, Key Tap, and Mow.

Of note, IMU-based wearable devices allow extraction of the features in time domain, frequency domain, time-frequency domain, and other based on the specific application [18].

The time-domain features are the general statistical features of a signal. The ones that are often used are mean, standard deviation, variance, mean absolute deviation (MAD), root mean square (RMS), median, zero crossing, interquartile range (IQR), correlation between axis, entropy, and kurtosis [45] [68]. These features typically play an essential role in movement classification and detection tasks if most discriminative features are not known.

The frequency domain signal provides frequency patterns of different activities. The frequency domain features analyze the frequency performance of the sensor signals, which is usually the periodicity of the signal over a long duration. Typical frequency domain features are the Fourier Transform, the spectral energy, and the Discrete Cosine Transform [45] [68]. Some of these features also can be employed when IMUs are used for activity recognition.

The study of Zhang et al. [80] showed the potential of IMU to access the motor disorder of upper limbs in PwPD and stroke patients. Objective assessment of upper limbs for post-stroke patients with the dynamic time wrapping signal from the $\mathrm{x}, \mathrm{y}, \mathrm{z}$ axis was estimated. The placement of the wireless IMU sensor was on the affected hand wrist. A novel single-index-based assessment approach for quantitative upperlimb mobility evaluation was proposed for post-stroke rehabilitation. Similarly, in 2013 Cavallo et al. [22] used the SensHand device to access the PwPD patients tremors. To evaluate the severity of the disease, different biomechanical parameters were evaluated from the signals coming from the accelerometers and the gyroscopes. In particular, according to the different exercises performed by the patients, the following features were extracted by Rovini et al. [67] using the SensHand: movement frequency, movement amplitude, movement velocity, variability of the movement in frequency and amplitude, energy expenditure, signal power, fundamental frequency, 
and percentage of power band. The results of the preliminary study [55] suggest a strong correlation with some biomechanical features and the clinical scale, thus providing an objective assessment of the disease.

\subsubsection{Features Selections}

Large-scale and high-dimensional data acquired from these wearable sensors required conversion of this data to meaningful information. Typically, these high dimensionalities are associated with high levels of noise. The two main causes of noise are imperfection of technology that collected the data and the sources of the data itself [71]. Feature selection procedures select a subset of features from the original feature set without any transformation, maintaing the physical meanings of the original features and selecting those features that are capable of discriminating between the samples that belong to different classes.

Three main approaches are used for feature selection: filter, wrapper, and embedded. Filter methods use statistical feature properties to filter out irrelevant attributes. Wrapper methods explore the entire attribute space to score subsets of attributes based on their predictive power. Embedded methods attempt to find an optimal subset of features while constructing the predictive model at the same time [51].

Dimensionality reduction is one of the most popular techniques. It is used to select the features with less dimensionality, thus to improve the machine learning classifier generalization ability and accuracy while lowering the computational complexity. Principal Component Analysis (PCA) is a popular feature selection method in terms of dimensionality reduction. PCA is a linear combination of all the original variables; thus it is often difficult to interpret the results [81]. Alternatively, lasso is a promising variable selection technique. It minimizes the residual sum of squares subject to the sum of the absolute value of the coefficients being less than a constant. Because of its nature, this constraint tends to produce some coefficients that are exactly 0 and hence gives interpretable sparse models which make it favorable as a variable selection method [73]. Another feature selection method is the KruskalWallis test. It is a non-parametric one-way analysis of variance (ANOVA) test that is simple to implement and widely used in clinical data sets [13]. Another feature selection method is the linear mixed effect model (LME), which is a powerful and flexible tool used to reduce the feature set when we need to differentiate patients on a clinical scale. The LME models are based on a restricted maximum likelihood estimation method and have been widely used in medical diagnostics studies [77]. Other feature selection methods are Pearson and Spearman correlations, which are often used to assess the correlation between two variables. Pearsons correlation assesses the linear relationship, whereas Spearmans correlation assesses the monotonic relationship between variables. Chi-square also is one of the most effective methods for feature selection; it measures the degree of independence between the feature and the categories. It also is quite effective when the number of features is very small [32]. 


\subsubsection{Classification Algorithms}

The main objective of the machine learning algorithms or classifications is to create patterns to describe, analyze and predict data. These patterns are created from a set of examples, instances, composed of the extracted features [45]. Generally, there are three different kinds of machine learning algorithms: supervised, unsupervised, and reinforcement learning.

In supervised machine learning algorithms, the training of the pattern recognition systems is made by a set of labeled data; in unsupervised machine learning, the system is trained with unlabeled data. In reinforcement learning, the machine is trained to make a specific decision. The machine learns from past decisions and tries to determine the best possible solution according to the available and past knowledge [28]. Different machine learning algorithms are used in the area of AAL applications. Some commonly used algorithms are thresholding-based classifiers, neural networks, support vector machine (SVM), hidden Markov model, instance-based (K-nearest-neighbors), and probabilistic (Nave Bayes (NB)).

Thresholding-based classifiers are often used in assisted living applications for the binary classification problem. They classify one of the two states based on the threshold. When the value of features is above the threshold, it will consider one state, and for feature value below the threshold, it is for the other state. Among these classifiers are decision trees (DT) and random forest (RF). Decision trees are widely used in recognition problems and are based on test questions and conditions. Each node represents a test; the branches represent outcomes, while the leaves are the final labels [45]. A random forest is made of a certain number of decision trees. Each tree gives a classification output, and the final output will be the maximum voting among the trees. Each tree is built on a vector of features built randomly from the starting ones [30].

Instance-based classifiers such as K-nearest neighbors make decisions based on the instance under test. These classifiers do not need training data, but they are computationally intensive.

Neural networks are basically human brain inspired classifiers generally used in deep learning for complex daily activities and also for clinical decisionmaking such as healthy or unhealthy. It consists of a large set of nodes with weighted connections. It can provide high classification accuracy with a large set of training data. However, typically in assisted living applications, a large set of training data often is not available, which make it less favorable in the assisted living applications [18].

SVM is a very popular classification algorithm often used in assisted living applications. SVM is unique among the other machine learning techniques because of its ability to distribute population in high-dimensional feature space. This algorithm was initially applied only to binary classification, finding the optimal separating hyperplane between two datasets. In a multiclass problem, a one-versus-one strategy is used to adopt SVM in multiclass problems [17]. SVM categorized the data based on the trained model by using a unique technique known as kernel. Different kinds of kernels can be found in advanced systems, including linear, polynomial, and radial 
basis function [63]. SVM does not need a large set for the training model, which is an advantage compared to neural networks [18].

HMM is the statistical Markov model, in which the system assumes the Markov process with unobserved hidden states. HMM can be presented as the simplest dynamic Bayesian network. HMM is often used in speech recognition and gesture recognition. It is commonly used in inertial measurement unit-based sensor data to recognize the daily activities and sequence of movements [18].

To compare the performances of the different classification algorithms and have a quantitative analysis of the results, different parameters can be evaluated beginning with the confusion matrix (i.e. accuracy, F-measure, precision, recall, and specificity, as described in [45]).

\subsection{Use cases: two applications of SensHand}

This section presents two concrete applications of gesture recognition based on wearable IMUs. The first application monitor daily activities to support senior citizens in maintaining control over their lives. The second application aims to provide remote decision support to clinicians. By means of the wearable sensors, the doctor could remotely monitor a PwPD patience to quantitatively evaluate the performance of a set of hand gestures.

\subsubsection{SensHand for recognizing daily Gesture}

In this application, the SensHand was used to recognize nine different gestures typically performed in activities of daily living (eat some crackers with the hand (HA); drink from a glass (GL); eat some fruit with the fork $(\mathrm{FK})$; eat some yogurt with a spoon (SP); drink from a cup (CP); answer the phone (PH); brush the teeth with a tooth brush (TB); brush the hair with a hair brush (HB); dry the hair with the hair dryer (HD)). The study consisted of different phases: in the first step, the attention was focused on finding the best combination of sensors that could recognize the gestures [57]. Then, the optimal solution was analyzed in greater depth to reduce the number of features to be used and use the new dataset as input for the machine learning algorithms.

\section{Experimental setting}

The SensHand was used for the acquisition, but the finger sensors were placed on the intermediate phalange of the index and middle finger instead of on the distal one. Data from accelerometers and gyroscopes were collected at $50 \mathrm{~Hz}$, already filtered with a fourth-order low-pass digital filter with a cutoff frequency of $5 \mathrm{~Hz}$. A more detailed description of the acquisition protocol can be found in [57]. .

\section{Data Processing and Analysis}

After the acquisition, the signals coming from the sensors were segmented according to the label to separate each gesture in the sequences. The complete dataset consisted of 7200 gestures. Mean, standard deviation, root mean square, and MAD were extracted from each axis of the accelerometer. 
As described in Moschetti et al. [57], two different machine learning algorithms, i.e. DT and SVM, were applied to find the best combination of sensors able to recognize the gestures. Two kinds of analysis were carried out, a personal analysis, where the same user was used as training and testing, and a leave-one-subject-out analysis (LOSO), where one participant was used as a test set on a model trained on the 19 participants. Results showed that the combination of sensors that use the index finger and the wrist sensors gave good results compared to other configurations. Using these two sensors provided excellent results in terms of accuracy and F-measure ( 0.89 and 0.884 , respectively) while maintaining a low obtrusiveness for the users.

Starting from this configuration, further analyses were conducted. In particular, the data set was reduced. Considering the sensor on the wrist and the one on the index finger, our dataset was made of 7200 gestures and 24 features. To remove the features that were too correlated, the Pearson correlation coefficient between each feature was computed, and the features with a coefficient greater than 0.85 were removed [15].

The new dataset is composed of 15 uncorrelated features. The matrix of the features was then normalized according to a Z-norm, to have zero mean and a unit standard deviation, to avoid distortion due to data heterogeneity.

To find similarity between gestures, for each gesture the mean values and the standard deviation were computed beginning with the reduced dataset. Then, PCA was applied to improve the visualization of the features in the principal component space. The standard deviation quantifies the variation in performing a specific gesture.

A SVM with a third-order polynomial kernel was applied to the features dataset to recognize the gestures and provide a quantitative analysis of the performances. A LOSO analysis was carried out to evaluate whether the system trained on 19 users was able to recognize the gestures performed by an unknown user. The system was trained leaving one user out, in turn, and then tested on the left-out subject. Then, a mean of the results was evaluated. This analysis was performed in Weka - Data Mining Suite [36].

\section{Results}

Results from previous work showed that using two sensors placed on the intermediate phalange of the finger and on the wrist allow recognition among nine gestures of daily living [57]. In this further analysis, a reduction of the features was computed, and a third-order polynomial kernel SVM was applied in a LOSO analysis to evaluate the performances of the system. Precision, recall, specificity, accuracy and F-measure were computed to have a quantitative analysis of the performance.

The analysis showed good results in terms of accuracy and F-measure $(0.9232$ \pm 0.05 and $0.9288 \pm 0.05$, respectively).

In Fig. 1.4 the values for the precision, recall and F-measure are reported for each gesture.

Considering the F-measure of each gesture, FK and HB are the worst recognized gestures. Looking at the confusion matrix (Fig. 1.5), it can be seen that the FK gesture is often confused with the SP one. The two gestures are often confused between each other due to the similarity of the movements. Other two gestures 


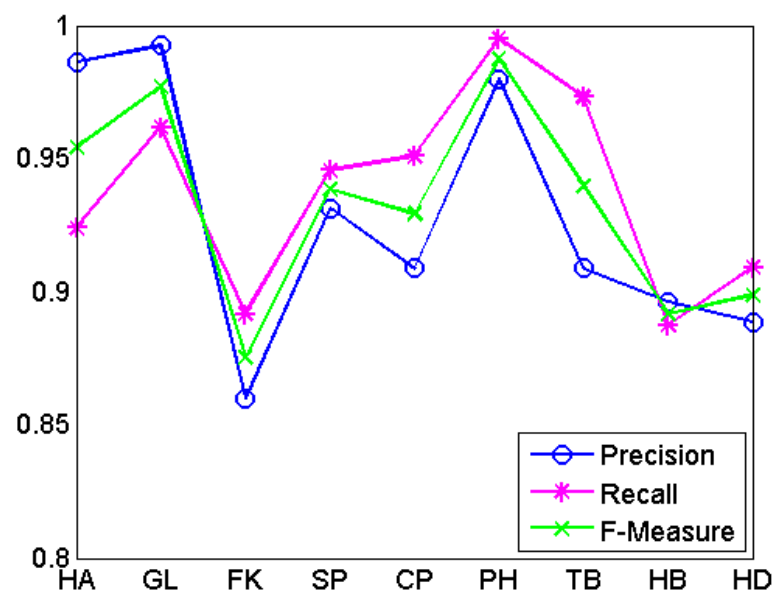

Figure 1.4: Values of precision, recall, and F-measure for each gesture

with a low F-measure with respect to the others are HB and HD. Even in this case, the confusion matrix shows how the two gestures are often mutually confused. In addition, the CP gesture does not reach a high value of F-measure, as it is often confused with GL. The highest values of F-measure are reached for HA, GL, and PH (all $>0.94$ ).

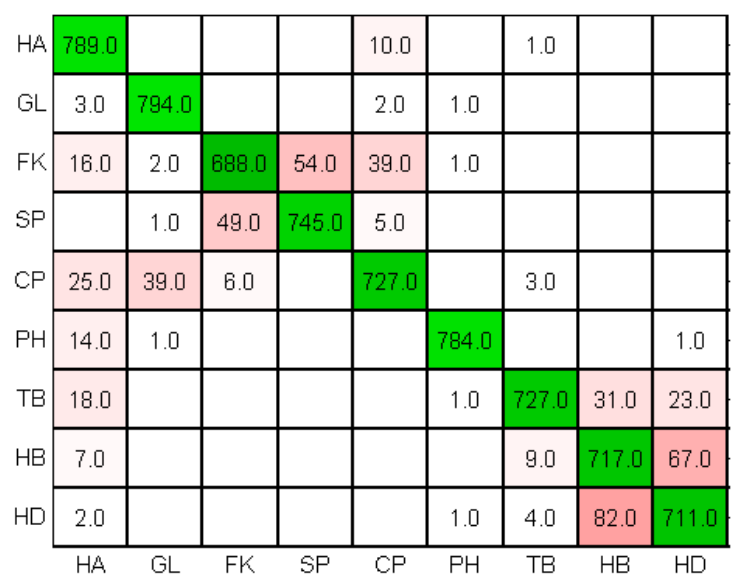

Figure 1.5: Confusion matrix for SVM model in the LOSO analysis for the entire dataset 
The same can be observed from the representation of the mean gestures in the PCA-space (Fig 1.6). In accordance with the variance, the first two principal components explain the $78.25 \%$ of the variance of the original dataset. HD and HB; $\mathrm{PH}$ and TB; CP,GL and HA; are very similar gestures, and sometimes they could be mutually confused, as shown in the confusion matrix. In Fig. 1.6, it is possible also to observe how similarly the users performed the gestures. As a matter of fact, the circle around the star represents the standard deviation from the mean value representing the gestures. It can be seen that the SP gesture is the most precise gesture performed by the 20 users; the standard deviation has the lower values. On the contrary, FK and PH are the gestures which have greater variability, meaning that the users had performed the gestures in different ways.

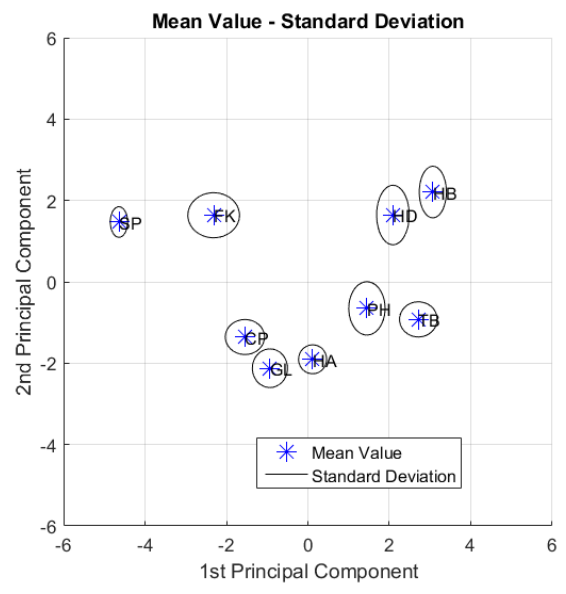

Figure 1.6: Mean gestures in the PCA-space.

These results showed that using a sensor on the index finger and a sensor on the wrist allows to recognize nine different gestures generally performed in activities of daily living. Recognizing these gestures could be useful to monitor persons during daily life, without being too obtrusive. As a matter of fact, at the moment, the SensHand was used to acquire the data, but further improvement could bring the development of a smart ring and smart bracelets, therefore decreasing the intrusiveness. The use of the same sensors could be further investigated to increase the number of gestures and thus activities to be recognized, and it could also be used to control robots and smart appliances.

\subsubsection{SensHand for supporting clinical decisions}

Gesture-based systems could be used to monitor the clinical status of the patients, providing a supporting tool for the clinicians. In this context, this use case aims to describe the development and evaluation methods for quantitative and automatic scoring of the hand motor performance of PwPD. In this study, two different group 
of subjects were involved. The first group included both healthy subjects and PwPD, whereas the second group included only PwPD subjects. This group was further divided into two sub-groups based on the average score obtained from MDS-UPDRS III scale given by clinicians across each exercise. The PwPD subjects belonging to scale 0 or 1 according to averaged rated scale were merged in one group named Slight-Mild (SM). The subjects with PwPD belonging to average rated scale 2 and 3 were merged in another group named Moderate-Severe (MS). The analysis is based on the standard supervised and unsupervised feature selection and classification techniques.

Examination of literature reveals a large number of techniques for the automatic detection of PwPD motor symptoms. However, generally machine learning algorithms were used to detect a single motor symptom as described in [11]. Typically, patients are likely to experience multiple symptoms, thus increasing the chance of false positive and false negative. Current study has been therefore focused on the multiple motor symptoms to improve the accuracy of machine learning algorithms to classify the PwPD on a clinical scale. The methodology carried out in this study is shown in Fig. 1.7 .

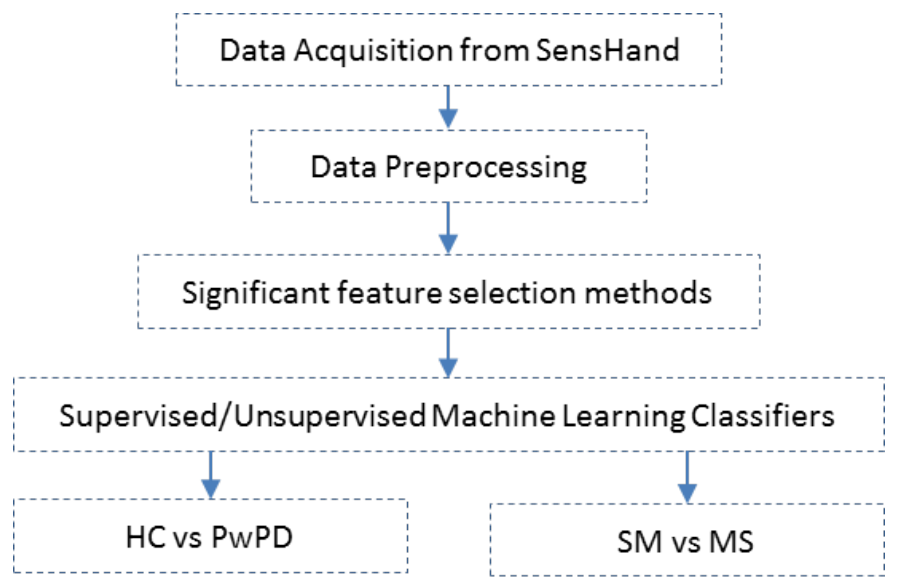

Figure 1.7: Flowchart showing classification methodology

\section{Data acquisition}

A total of 115 subjects were involved in the study after being evaluated by a neurologist by means of MDS-UPDRS III, for a general evaluation of the disease.

The first group was composed of 28 PwPD patients (18 men, 10 women; mean age \pm SD, $66.2 \pm 10.2$ years old; MDS-UPDRS III score: $14.5 \pm 7.5$; Hoehn Yahr (HY) score: $1.74 \pm 0.6$; Levodopa Equivalent Dose: $407.2 \mathrm{mg} \pm 311.5)$ and 28 healthy subjects (HC) (22 men, 6 women, mean age SD, 65.02 .7 years old.

The second group was composed of 59 PwPD patients (42 men, 19 women; mean age $\pm \mathrm{SD}, 67.3 \pm 8.8$ years old). All patients were on their medication before and during the experiments. 
All these subjects lived independently in the community and gave written informed consent prior to the study. The studies' procedures were approved by the Medical Ethical Committee of ASL1 (Azienda Sanitaria Locale, Massa and Carrara, Italy; approval n1148/12.10.10).

According to the neurologist and to the tasks required in the motor section of the MDS-UPDRS (MDS-UPDRS III), an experimental protocol comprised of six exercises has been proposed to analyze the motor skills of the upper limbs of the participants [55]. The selected exercises are: Thumb-Forefinger Tapping (THFF), Hand Opening/Closing (OPCL), Forearm Pronation/Supination (PSUP), Resting Tremor (REST). The protocol and the processing of the data are described in [67].

\section{Features Section}

The features extracted from the SensHand are the biomechanical features described in [67]. To select the most significant features and to investigate the discriminative ability of the parameters, two methods were used, as mentioned above: Lasso, Kruskal-Wallis. Parameters which satisfied at least one feature selection criteria, as mentioned above, was considered as a statistically significant parameter. Table 1.1 shows the features which satisfied the feature selection criteria.

\begin{tabular}{llll}
\hline Exercise & Features & Lasso & Kruskall-Wallis \\
\hline \multirow{2}{*}{ THFF } & Opening velocity & 9.568 & 0.0052 \\
& Closing velocity & 2.793 & 0.0028 \\
\hline \multirow{2}{*}{ OPCL } & Number of movements & 0.021 & 0.0001 \\
& Opening velocity & 5.702 & 0.0002 \\
& Closing velocity & 6.677 & 2.3019 \\
\multirow{2}{*}{ REST } & Power in tremor frequency & 8.273 & 0.0038 \\
& band from accelerometer & & \\
& signal & & \\
& Power in tremor frequency & 1.662 & \\
& band [3.5-7.5] from gyro- & & \\
& scope signal & & \\
&
\end{tabular}

Table 1.1: Most significant features extracted.THFF is the ThumbForefinger Tapping exercise, OPCL is the Hand Opening-Closing exercise, and REST is the Rest Tremor exercise [67]. A detailed list of the parameters is reported in Appendix

Results To classify the healthy and PwPD individuals, multiple supervised machine learning classifiers was used. To train the classifiers, selected significant features were paired with each other randomly to obtain the maximum classification accuracy. To minimize the subjective effect in training model, leave-one-subject-out cross validation has been used. SVM was used with sequential minimum optimization (SMO) [40] algorithm used to support the kernel function to make the linear boundary with the linear kernel.

Regarding the comparison between HC and PwPD, the maximum $83.63 \%$ accuracy was obtained from the SVM with the following paired features: opening 
velocity(degs/s) of the forefinger, closing velocity of the forefinger, power in tremor frequency band [3.5-7.5] from accelerometer signal, power in tremor frequency band [3.5-7.5] from gyroscope signal (see Fig 1.8].

\section{Classification Test : SVM}

Total Instances $=55$

Correctly Classified $=46$

Incorrectly classified $=9$

AUC $=86 \%$

\begin{tabular}{|l|l|l|}
\hline & PwPD & HC \\
\hline PwPD & 21 & 4 \\
\hline HC & 5 & 25 \\
\hline
\end{tabular}

Receiver operating characteristics curves b/w Healthy and

\begin{tabular}{|l|l|}
\hline Sensitivity & $85 \%$ \\
\hline Specificity & $83 \%$ \\
\hline Accuracy & $83.63 \%$ \\
\hline
\end{tabular}

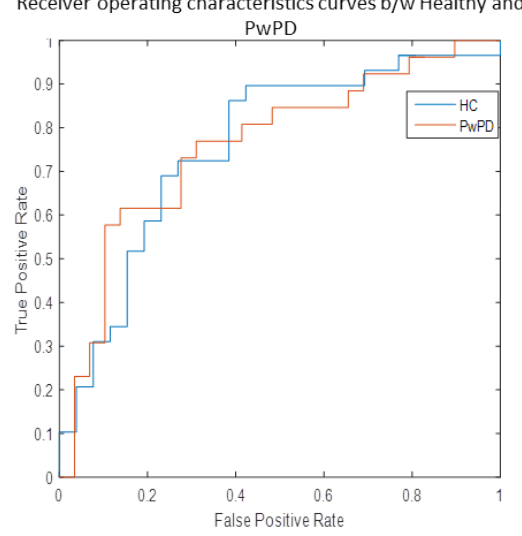

Figure 1.8: Classification between PwPD and healthy control with SVM

Two different supervised classification methods, SVM and logistic regression, were used to classify the SM and MS subjects. LOSO cross validation was adopted to avoid the subjective effect on the training dataset. Most significant features paired with each other randomly to obtain the maximum classification accuracy. Maximum classification accuracy was obtained from four paired features: opening hand velocity, opening closing number of movements of hand, power in the tremor frequency band [3.5-7.5] from accelerometer signal, power in tremor frequency band [3.5-7.5] from gyroscope signal.

As reported in Fig. 1.9, the best performing method was the SVM classifier, which classified SM and MS groups with an accuracy of 79.66\%, sensitivity of 94\%, and specificity of $52 \%$, respectively. Low specificity directs the highest variability in slight and moderate patients samples due to highest severity level of the disease.

In this study, we proposed a method for quantifying PwPD motor symptoms in both initial and advanced patients experiencing motor fluctuations. The symptoms are quantified by calculating several biomechanical parameters from motor exercises for upper limbs by device sensHandv1. Different standard machine learning classifiers, the support vector machine, and logistic regression were used.

Overall, the method had good test reliability and provided high discriminating power between PwPD and control subjects with the 83\% classification accuracy. Similarly, for the SM and MS group classification, the $80 \%$ classification accuracy was obtained. These results not only endorse the ability of sensHandV 1 to assess the biomechanical parameters accurately from the upper limb exercises, but also suggest that these biomechanical parameters have the potential to discriminate the PwPD on a clinical scale. 
Classification Test

\begin{tabular}{|c|c|c|c|c|c|}
\hline \multicolumn{3}{|c|}{$\begin{array}{l}\quad \text { Logistic Regression } \\
\text { Total Instances }=58 \\
\text { Correctly Classified }=45 \\
\text { Incorrectly classified }=13 \\
\text { AUC }=71.93 \%\end{array}$} & \multicolumn{3}{|c|}{$\begin{array}{l}\quad \text { SVM } \\
\text { Total Instances }=59 \\
\text { Correctly Classified }=47 \\
\text { Incorrectly classified }=12 \\
\text { AUC }=80 \%\end{array}$} \\
\hline & SM & MS & & SM & MS \\
\hline SM & 35 & 3 & SM & 21 & 4 \\
\hline MS & 11 & 10 & MS & 5 & 25 \\
\hline \multicolumn{2}{|c|}{ Sensitivity } & $92 \%$ & \multicolumn{2}{|c|}{ Sensitivity } & $94 \%$ \\
\hline \multicolumn{2}{|c|}{ Specificity } & $47 \%$ & \multicolumn{2}{|c|}{ Specificity } & $52 \%$ \\
\hline \multicolumn{2}{|c|}{ Accuracy } & $77.66 \%$ & \multicolumn{2}{|c|}{\begin{tabular}{|l} 
Accuracy \\
\end{tabular}} & $79.66 \%$ \\
\hline
\end{tabular}

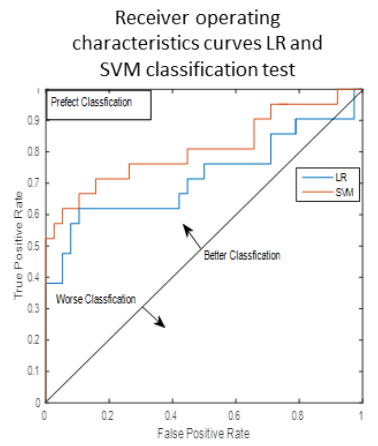

Figure 1.9: Classification between Slight-Mild (SM) and Moderate-Severe (MS) groups with Logistic Regression and SVM

In the previous studies [11], the lack of representative measures was an important limitation in the objective measuring system, as most of the multi-dimensional motions are presented in the form of separate physical parameters. At the same time, none of the individual parameters would be representative to evaluate the motor performance of the upper and lower limbs. Therefore, in the future, an integrated and comprehensive matrix is needed to make a gold standard for the assessment of motor performance for the upper and lower limbs to evaluate the PwPD subjects. Additionally, the fusion of data acquired from different body parts seems to be the most logical approach to improve the accuracy of biomechanical parameters and assessment of PD as presented in the PERFORM system [74].

\subsection{Conclusion and brief roadmap}

According to the demographic projection, the number of elderly people will increase in the coming years, raising challenges to find new solutions to help them stay healthy and independent. AAL technologies can provide solutions to help older individuals stay longer in their own homes, monitored and supported, and to make them and their caregivers feel safe. As described in Section 1.3, gesture recognition can play an important role in the use of this technological solution.

In recent years, various technologies have been developed to recognize gestures, spanning from wearable sensors to video-based sensors. The choice of the device strongly depends on the application. In this chapter, two types of sensors for gesture recognition have been presented: SensHand and LeapMotion. The first is a wearable device based on inertial measurement units, while the second is a vision-based sensor. Both of them showed good results in the applications where they were used, in particular for recognition of daily gestures (SensHand) and in the objective evalua- 
tion of PwPD (SensHand and LeapMotion), showing that the same device could be used for different aims.

Considering the presented applications, a few steps remain to bring these technologies into real life. In particular, regarding use of the SensHand for recognition of daily gestures, the next steps will be to implement an online algorithm that will allow the sensor to be used in daily living, and be able to discriminate among different gestures. Moreover, other applications could be implemented so it can be used for training purposes as well. In addition, regarding LeapMotion, further studies should be conducted to perform all proposed clinical assessment protocols for motor disorders in PwPD. Moreover, a tutorial for the patients is needed to show them where to place their hand during the data acquisition. People can indeed perform exercises with this sensor while being at home, which will create a continuum of care and increase the rehabilitation hours, making it more efficient. However, further investigations should be performed to determine the most comfortable way to use the different technologies for all the patients, with low to advanced stages of disease.

Future developments will look toward the implementation of different applications using the same sensors. The use of a set of sensors at home for monitoring, training, and controlling smart appliances and devices would allow individuals to have a set of tools to be used in daily life. In a house with many AAL technologies, for instance, robots could be controlled and teleoperated to make them perform dangerous activities such as preventing people from falling and becoming injured. The same sensors could be used by the robot to perceive what the user is doing and the understand the non-verbal cues that people use to communicate, allowing a more natural interaction. At the same time, sensors can be used as monitoring tools both for clinicians in case of already existing diseases and also for checking daily habits and preventing degeneration of cognitive and physical abilities. Therefore, gesture recognition represents an important tool for several AAL applications. 


\section{Bibliography}

[1] Ambient Assisted Living Roadmap. http://www.aaliance2.eu/ sites/default/files/AA2_WP2_D2\%207_RM2_rev5.0.pdf Accessed: 2016-11-21.

[2] Ambient Assisted Living Strategic Research Agenda. http: //wWw.aaliance2.eu/sites/default/files/AA2_D2.7b_ SRA2014_v4.0.pdf. Accessed: 2016-11-21.

[3] CSS Insight - Wearable Tech Market To Be Worth \$34 Billion By 2019. http://www.ccsinsight.com/press/company-news/ 2332-wearables-market-to-be-worth-25-billion-by-2019-reveal Accessed: 2016-11-24.

[4] Demography - trends and projections 2015, European Commission. http://ec.europa.eu/economy_finance/structural_ reforms/ageing/demography/index_en.htm. Accessed: 2016-1121.

[5] Ericsson Mobility Report -2016. https://www.ericsson.com/res/ docs/2016/ericsson-mobility-report-2016.pdf. Accessed: 2016-11-24.

[6] Medical Electronics Market - Global Forecast to 2022. http: //www.marketsandmarkets.com/PressReleases / medical-electronics.asp. Accessed: 2016-11-21.

[7] Smart Home Market - Global Forecast to 2022. http: //www.marketsandmarkets.com/Market-Reports/ smart-homes-and-assisted-living-advanced-technologie-and-gl html. Accessed: 2016-11-21.

[8] Telehealth Market - Global Forecast to 2022. http://www. marketsandmarkets.com/PressReleases/telehealth.asp. Accessed: 2016-11-21.

[9] The advent of digital health. http://www.strategy-business.com/ blog/The-Advent-of-Digital-Health?gko=f2f63. Accessed: 2016-11-21. 
[10] Vijayalakshmi Ahanathapillai, James D Amor, Zoe Goodwin, and Christopher J James. Preliminary study on activity monitoring using an android smartwatch. Healthcare technology letters, 2(1):34-39, 2015.

[11] Claas Ahlrichs and Michael Lawo. Parkinson's disease motor symptoms in machine learning: A review. arXiv preprint arXiv:1312.3825, 2013.

[12] Carmen Alabart Gutiérrez del Olmo. Study and analysis of the leap motion sensor and the software development kit (sdk) for the implementation of visual human computer interfaces. 2015.

[13] Sajid Ali Khan, Ayyaz Hussain, Abdul Basit, and Sheeraz Akram. Kruskalwallis-based computationally efficient feature selection for face recognition. The Scientific World Journal, 2014, 2014.

[14] Christoph Amma, Marcus Georgi, and Tanja Schultz. Airwriting: Hands-free mobile text input by spotting and continuous recognition of 3d-space handwriting with inertial sensors. In 2012 16th International Symposium on Wearable Computers, pages 52-59. IEEE, 2012.

[15] Ferhat Attal, Samer Mohammed, Mariam Dedabrishvili, Faicel Chamroukhi, Latifa Oukhellou, and Yacine Amirat. Physical human activity recognition using wearable sensors. Sensors, 15(12):31314-31338, 2015.

[16] David I Auerbach. Will the np workforce grow in the future?: New forecasts and implications for healthcare delivery. Medical care, 50(7):606-610, 2012.

[17] Rezaul Begg and Joarder Kamruzzaman. A machine learning approach for automated recognition of movement patterns using basic, kinetic and kinematic gait data. Journal of biomechanics, 38(3):401-408, 2005.

[18] Terrell R Bennett, Jian Wu, Nasser Kehtarnavaz, and Roozbeh Jafari. Inertial measurement unit-based wearable computers for assisted living applications: A signal processing perspective. IEEE Signal Processing Magazine, 33(2):28-35, 2016.

[19] Gerald Bieber, Marian Haescher, and Matthias Vahl. Sensor requirements for activity recognition on smart watches. In Proceedings of the 6th International Conference on PErvasive Technologies Related to Assistive Environments, page 67. ACM, 2013.

[20] The Duy Bui and Long Thang Nguyen. Recognizing postures in vietnamese sign language with mems accelerometers. IEEE sensors journal, 7(5):707-712, 2007.

[21] Nicola Carbonaro, Alberto Greco, Gaetano Anania, Gabriele Dalle Mura, Alessandro Tognetti, EP Scilingo, Danilo De Rossi, and Antonio Lanata. Unobtrusive physiological and gesture wearable acquisition system: a preliminary study on behavioral and emotional correlations. Global Health, pages 88-92, 2012. 
[22] Filippo Cavallo, Dario Esposito, Erika Rovini, Michela Aquilano, Maria Chiara Carrozza, Paolo Dario, Carlo Maremmani, and Paolo Bongioanni. Preliminary evaluation of senshand $\mathrm{v} 1$ in assessing motor skills performance in parkinson disease. In Rehabilitation Robotics (ICORR), 2013 IEEE International Conference on, pages 1-6. IEEE, 2013.

[23] Kai-Hsiang Chen, Po-Chieh Lin, Yu-Jung Chen, Bing-Shiang Yang, and ChinHsien Lin. Development of method for quantifying essential tremor using a small optical device. Journal of neuroscience methods, 266:78-83, 2016.

[24] Tushar Chouhan, Ankit Panse, Anvesh Kumar Voona, and SM Sameer. Smart glove with gesture recognition ability for the hearing and speech impaired. In Global Humanitarian Technology Conference-South Asia Satellite (GHTCSAS), 2014 IEEE, pages 105-110. IEEE, 2014.

[25] Diane J Cook and Narayanan C Krishnan. Activity Learning: Discovering, Recognizing, and Predicting Human Behavior from Sensor Data. John Wiley \& Sons, 2015.

[26] Sebastian Deterding, Dan Dixon, Rilla Khaled, and Lennart Nacke. From game design elements to gamefulness: defining gamification. In Proceedings of the 15th international academic MindTrek conference: Envisioning future media environments, pages 9-15. ACM, 2011.

[27] Laura Dipietro, Angelo M Sabatini, and Paolo Dario. A survey of glove-based systems and their applications. IEEE Transactions on Systems, Man, and Cybernetics, Part C (Applications and Reviews), 38(4):461-482, 2008.

[28] Charles Elkan. Evaluating classifiers. 2012.

[29] Dario Esposito and Filippo Cavallo. Preliminary design issues for inertial rings in ambient assisted living applications. In 2015 IEEE International Instrumentation and Measurement Technology Conference (I2MTC) Proceedings, pages 250-255. IEEE, 2015.

[30] Zengtao Feng, Lingfei Mo, and Meng Li. A random forest-based ensemble method for activity recognition. In 2015 37th Annual International Conference of the IEEE Engineering in Medicine and Biology Society (EMBC), pages 5074-5077. IEEE, 2015.

[31] A Ferrone, F Maita, L Maiolo, M Arquilla, A Castiello, A Pecora, X Jiang, $\mathrm{C}$ Menon, and L Colace. Wearable band for hand gesture recognition based on strain sensors. In Biomedical Robotics and Biomechatronics (BioRob), 2016 6th IEEE International Conference on, pages 1319-1322. IEEE, 2016.

[32] Xiubo Geng, Tie-Yan Liu, Tao Qin, and Hang Li. Feature selection for ranking. In Proceedings of the 30th annual international ACM SIGIR conference on Research and development in information retrieval, pages 407-414. ACM, 2007. 
[33] Marcus Georgi, Christoph Amma, and Tanja Schultz. Recognizing hand and finger gestures with imu based motion and emg based muscle activity sensing. In Proceedings of the International Conference on Bio-inspired Systems and Signal Processing, pages 99-108, 2015.

[34] Sarah L Gorst, Christopher J Armitage, Simon Brownsell, and Mark S Hawley. Home telehealth uptake and continued use among heart failure and chronic obstructive pulmonary disease patients: a systematic review. Annals of Behavioral Medicine, 48(3):323-336, 2014.

[35] Marc Gowing, Amin Ahmadi, François Destelle, David S Monaghan, Noel E OConnor, and Kieran Moran. Kinect vs. low-cost inertial sensing for gesture recognition. pages 484-495, 2014.

[36] Mark Hall, Eibe Frank, Geoffrey Holmes, Bernhard Pfahringer, Peter Reutemann, and Ian H Witten. The weka data mining software: an update. ACM SIGKDD explorations newsletter, 11(1):10-18, 2009.

[37] Roderick S Hooker, James F Cawley, and Christine M Everett. Predictive modeling the physician assistant supply: 2010-2025. Public health reports, pages 708-716, 2011.

[38] Nastaran Hosseinpour. Ict in health-care. 2016.

[39] Mi-Chia Hsieh, Yu-Hua Yen, and Tsung-Ying Sun. Gesture recognition with two 3-axis accelerometers. In Consumer Electronics-Taiwan (ICCE-TW), 2014 IEEE International Conference on, pages 239-240. IEEE, 2014.

[40] Chih-Wei Hsu, Chih-Chung Chang, Chih-Jen Lin, et al. A practical guide to support vector classification. 2003.

[41] Shuai Jin, Yi Li, Guang-ming Lu, Jian-xun Luo, Wei-dong Chen, and Xiaoxiang Zheng. Som-based hand gesture recognition for virtual interactions. In VR Innovation (ISVRI), 2011 IEEE International Symposium on, pages 317322. IEEE, 2011.

[42] Lei Jing, Yinghui Zhou, Zixue Cheng, and Tongjun Huang. Magic ring: A finger-worn device for multiple appliances control using static finger gestures. Sensors, 12(5):5775-5790, 2012.

[43] Pyeong-Gook Jung, Gukchan Lim, Seonghyok Kim, and Kyoungchul Kong. A wearable gesture recognition device for detecting muscular activities based on air-pressure sensors. IEEE Transactions on Industrial Informatics, 11(2):485494, 2015.

[44] Ji-Hwan Kim, Nguyen Duc Thang, and Tae-Seong Kim. 3-d hand motion tracking and gesture recognition using a data glove. In 2009 IEEE International Symposium on Industrial Electronics, pages 1013-1018. IEEE, 2009. 
[45] Oscar D Lara and Miguel A Labrador. A survey on human activity recognition using wearable sensors. IEEE Communications Surveys \& Tutorials, 15(3):1192-1209, 2013.

[46] JING Lei, ZHOU Yinghui, Zixue Cheng, and WANG Junbo. A recognition method for one-stroke finger gestures using a mems $3 \mathrm{~d}$ accelerometer. IEICE transactions on information and systems, 94(5):1062-1072, 2011.

[47] Jhe-Wei Lin, Chiuan Wang, Yi Yao Huang, Kuan-Ting Chou, Hsuan-Yu Chen, Wei-Luan Tseng, and Mike Y Chen. Backhand: Sensing hand gestures via back of the hand. In Proceedings of the 28th Annual ACM Symposium on User Interface Software \& Technology, pages 557-564. ACM, 2015.

[48] Kui Liu, Chen Chen, Roozbeh Jafari, and Nasser Kehtarnavaz. Multi-hmm classification for hand gesture recognition using two differing modality sensors. In Circuits and Systems Conference (DCAS), 2014 IEEE Dallas, pages 1-4. IEEE, 2014.

[49] Wei Lu, Zheng Tong, and Jinghui Chu. Dynamic hand gesture recognition with leap motion controller. IEEE Signal Processing Letters, 23(9):1188-1192, 2016.

[50] Wei Lu, Zheng Tong, and Jinghui Chu. Dynamic hand gesture recognition with leap motion controller. IEEE Signal Processing Letters, 23(9):1188-1192, 2016.

[51] Sebastián Maldonado, Ricardo Montoya, and Richard Weber. Advanced conjoint analysis using feature selection via support vector machines. European Journal of Operational Research, 241(2):564-574, 2015.

[52] Sushmita Mitra and Tinku Acharya. Gesture recognition: A survey. IEEE Transactions on Systems, Man, and Cybernetics, Part C (Applications and Reviews), 37(3):311-324, 2007.

[53] Mohamed A Mohandes. Recognition of two-handed arabic signs using the cyberglove. Arabian Journal for Science and Engineering, 38(3):669-677, 2013.

[54] A Moncada-Torres, K Leuenberger, R Gonzenbach, A Luft, and R Gassert. Activity classification based on inertial and barometric pressure sensors at different anatomical locations. Physiological measurement, 35(7):1245, 2014.

[55] Yosry S Morsi. Optimizing Assistive Technologies for Aging Populations. IGI Global, 2015.

[56] Alessandra Moschetti, Laura Fiorini, Michela Aquilano, Filippo Cavallo, and Paolo Dario. Preliminary findings of the aaliance 2 ambient assisted living roadmap. In Ambient Assisted Living, pages 335-342. Springer, 2014.

[57] Alessandra Moschetti, Laura Fiorini, Dario Esposito, Paolo Dario, and Filippo Cavallo. Recognition of daily gestures with wearable inertial rings and bracelets. Sensors, 16(8):1341, 2016. 
[58] Hossein Mousavi Hondori and Maryam Khademi. A review on technical and clinical impact of microsoft kinect on physical therapy and rehabilitation. Journal of medical engineering, 2014, 2014.

[59] Ganesh R Naik and Hung T Nguyen. Nonnegative matrix factorization for the identification of emg finger movements: evaluation using matrix analysis. IEEE journal of biomedical and health informatics, 19(2):478-485, 2015.

[60] John R Napier. The prehensile movements of the human hand. Bone \& Joint Journal, 38(4):902-913, 1956.

[61] Farid Parvini and Cyrus Shahabi. Utilizing bio-mechanical characteristics for user-independent gesture recognition. In 21st International Conference on Data Engineering Workshops (ICDEW'05), pages 1170-1170. IEEE, 2005.

[62] Jun Rekimoto. Gesturewrist and gesturepad: Unobtrusive wearable interaction devices. In Wearable Computers, 2001. Proceedings. Fifth International Symposium on, pages 21-27. IEEE, 2001.

[63] Gabriele Rescio, Alessandro Leone, and Pietro Siciliano. Supervised expert system for wearable mems accelerometer-based fall detector. Journal of Sensors, 2013, 2013.

[64] C Rishikanth, Harini Sekar, Gautham Rajagopal, Ramesh Rajesh, and Vineeth Vijayaraghavan. Low-cost intelligent gesture recognition engine for audiovocally impaired individuals. In Global Humanitarian Technology Conference (GHTC), 2014 IEEE, pages 628-634. IEEE, 2014.

[65] Mehran Roshandel, Aarti Munjal, Peyman Moghadam, Shahin Tajik, and Hamed Ketabdar. Multi-sensor based gestures recognition with a smart finger ring. In International Conference on Human-Computer Interaction, pages 316-324. Springer, 2014.

[66] Erika Rovini, Dario Esposito, Carlo Maremmani, Paolo Bongioanni, and Filippo Cavallo. Using wearable sensor systems for objective assessment of parkinsons disease. In 20th IMEKO TC4 International Symposium and 18th International Workshop on ADC Modelling and Testing Research on Electric and Electronic Measurement for the Economic Upturn, pages 15-17, 2014.

[67] Erika Rovini, Dario Esposito, Carlo Maremmani, Paolo Bongioanni, and Filippo Cavallo. Empowering patients in self-management of parkinsons disease through cooperative ict systems. Optimizing Assistive Technologies for Aging Populations, page 251, 2015.

[68] Muhammad Shoaib, Stephan Bosch, Ozlem Durmaz Incel, Hans Scholten, and Paul JM Havinga. Fusion of smartphone motion sensors for physical activity recognition. Sensors, 14(6):10146-10176, 2014. 
[69] Ionel Staretu and Catalin Moldovan. Leap motion device used to control a real anthropomorphic gripper. International Journal of Advanced Robotic Systems, 13(3):113, 2016.

[70] Nagender Kumar Suryadevara, Subhas C Mukhopadhyay, Ruili Wang, and RK Rayudu. Forecasting the behavior of an elderly using wireless sensors data in a smart home. Engineering Applications of Artificial Intelligence, 26(10):2641-2652, 2013.

[71] Jiliang Tang, Salem Alelyani, and Huan Liu. Feature selection for classification: A review. Data Classification: Algorithms and Applications, page 37, 2014.

[72] Rosanna Tarricone and Agis D Tsouros. Home care in Europe: the solid facts. WHO Regional Office Europe, 2008.

[73] Robert Tibshirani. Regression shrinkage and selection via the lasso. Journal of the Royal Statistical Society. Series B (Methodological), pages 267-288, 1996.

[74] Alexandros T Tzallas, Markos G Tsipouras, Georgios Rigas, Dimitrios G Tsalikakis, Evaggelos C Karvounis, Maria Chondrogiorgi, Fotis Psomadellis, Jorge Cancela, Matteo Pastorino, María Teresa Arredondo Waldmeyer, et al. Perform: A system for monitoring, assessment and management of patients with parkinsons disease. Sensors, 14(11):21329-21357, 2014.

[75] Ger van den Broek, Filippo Cavallo, and Christian Wehrmann. AALIANCE ambient assisted living roadmap, volume 6. IOS press, 2010.

[76] Daryl J Wile, Ranjit Ranawaya, and Zelma HT Kiss. Smart watch accelerometry for analysis and diagnosis of tremor. Journal of neuroscience methods, 230:1-4, 2014.

[77] Bodo Winter. Linear models and linear mixed effects models in r with linguistic applications. arXiv preprint arXiv:1308.5499, 2013.

[78] Michael T Wolf, Christopher Assad, Matthew T Vernacchia, Joshua Fromm, and Henna L Jethani. Gesture-based robot control with variable autonomy from the jpl biosleeve. In Robotics and Automation (ICRA), 2013 IEEE International Conference on, pages 1160-1165. IEEE, 2013.

[79] Haibin Yan, Marcelo H Ang Jr, and Aun Neow Poo. A survey on perception methods for human-robot interaction in social robots. International Journal of Social Robotics, 6(1):85-119, 2014.

[80] Zhe Zhang, Qiang Fang, and Xudong Gu. Objective assessment of upperlimb mobility for poststroke rehabilitation. IEEE Transactions on Biomedical Engineering, 63(4):859-868, 2016.

[81] Hui Zou, Trevor Hastie, and Robert Tibshirani. Sparse principal component analysis. Journal of computational and graphical statistics, 15(2):265-286, 2006. 